\title{
PRACTICE
}

\section{Eruptive xanthomas presenting in tattoos}

\author{
Hui Gao MD, Jing Chen MD
}
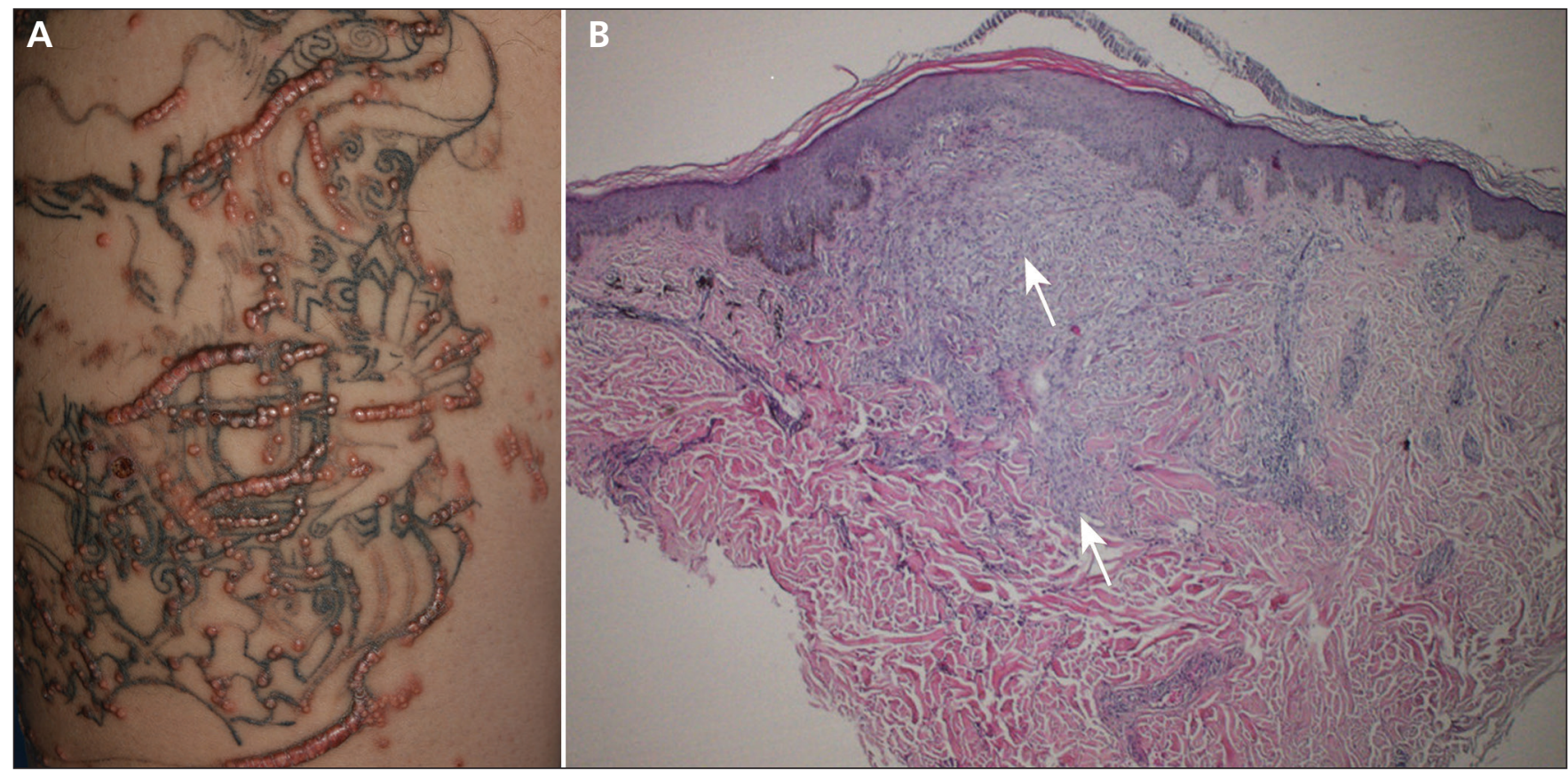

Figure 1: (A) Blue tattoos on the right leg of a 26-year-old man covered by a linear arrangement of xanthomatous papules. (B) Histology of the lesion (hematoxylin-eosin, original magnification $\times 40$ ) showing an essentially normal epidermis. There is infiltration of numerous nodular foam cells (arrows) and a few multinucleate giant cells within the dermis. Synthetic colours can be seen in the hair follicle and perifollicular tissues.

Competing interests: None declared.

This article has been peer reviewed.

The authors have obtained patient consent.

Affiliation: Department of Dermatology, Tianjin

Gongan Hospital, Tianjin, China

\section{Correspondence to:}

Hui Gao,

gaohuigongan@163.com

Acknowledgement: The authors acknowledge the assistance of Jia-chun Xu.

CMAJ 2015. DOI:10.1503 /cmaj.140383

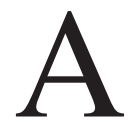
26-year-old man with a history of uncontrolled hyperlipidemia presented with a six-month history of painful, multiple papules on his chest, back and limbs. The two-year-old tattoos on his right leg were covered by a linear arrangement of papules, suggesting a Koebner phenomenon (Figure 1A). A biopsy from the lesions on the tattoos showed infiltration of nodular foam cells and a few multinucleate giant cells presenting within the dermis. Synthetic colours were observed in the hair follicle and the surrounding tissues (Figure 1B). Our patient had a triglyceride level of 4.52 (normal 0.24-1.70) $\mathrm{mmol} / \mathrm{L}$ and total cholesterol level of 10.5 (normal 2.8-5.7) mmol/ L. We diagnosed eruptive xanthomas and prescribed fenofibrate $200 \mathrm{mg} / \mathrm{d}$. After four weeks of treatment with fenofibrate, the patient's papules had not resolved.
The Koebner phenomenon refers to skin lesions occurring along lines of trauma. It has previously been reported in tattoos in conjunction with eruptive xanthoma and psoriasis. ${ }^{1-3}$ Our case is unusual in that the skin trauma occurred in the distant past, although at least two cases associated with remote skin trauma have been previously described. ${ }^{4}$ Our patient's tattoos were completed 18 months before the xanthomas appeared.

\section{References}

1. Miwa N, Kanzaki T. The Koebner phenomenon in eruptive xanthoma. J Dermatol 1992;19:48-50.

2. Goldstein GD. The Koebner response with eruptive xanthomas. J Am Acad Dermatol 1984;10:1064-5.

3. Arias-Santiago S, Espiñeira-Carmona MJ, Aneiros-Fernádez J. The Koebner phenomenon: psoriasis in tattoos. CMAJ 2013; 185:585.

4. Roederer G, Xhignesse M, Davignon J. Eruptive and tuberoeruptive xanthomas of the kin arising on sites of prior injury. Two case reports. JAMA 1988;260:1282-3. 